\title{
Comparison of spring oats varieties in response to the effects of root rot toxins in an in vitro culture
}

\author{
Zobova N.V.*, Lugovtsova S.Yu., Neshumaeva N.A. \\ Krasnoyarsk Research Institute of Agriculture, FRC KSC SB RAS, Krasnoyarsk, Russia \\ *e-mail: zobovnat@mail.ru
}

The effect of the Fusarium poae and Fusarium equiseti toxic metabolites was studied in vitro in oats immature embryos. The Murashige-Skoog medium (MS) and the classical 3-stage cultivation were used: induction, proliferation of calli and plant regeneration. Toxic metabolites obtained on the basis of isolates of the author's collection of regional pathogens on the nutrient medium Chapeka. Filtrates of toxic metabolites of the species $F$. poae and $F$. eqiseti were used as selective agents on the proliferation medium of calli in concentrations of $30 \%, 40 \%$ and $50 \%$. The volume of the material was 2578 immature embryos of eight varieties: Tubinsky, Sayan, Kazyr, Selma, Talisman, Zolotoi pochatok, Golets, Tyumen holozerny. Callus induction took place on MS with the addition of 2,4-D - $3 \mathrm{mg} / 1$ and IAA - $2 \mathrm{mg} / 1$, proliferation - from 2,4-D - $1.5 \mathrm{mg} / 1$, regeneration - with kinetin - $1 \mathrm{mg} / \mathrm{l}$ and IAA $-0.5 \mathrm{mg} / 1$. Inhibition of proliferation of calli by metabolites is highly significant even for lower concentrations of 30-40\% (from 49.4 to $71.0 \%$ for $F$. poae and $37.6-66.1 \%$ for $F$. eqiseti). Under these conditions the Tyumen holozerny and Kazyr varieties responded less to stress. Organogenesis and the formation of full-fledged regenerants in the presence of metabolites was above average for these same forms. Some dependence of the varietal reaction on the type of micromycetes was observed. The varieties Selma and Talisman compared with others showed greater resistance to the metabolites of $F$. eqiseti, and not of $F$. poae. In general, the metabolites of $F$. poae caused a stronger inhibitory effect on the stages of morphogenesis. Less resistant to the inhibitory effects of two types of micromycetes p. Fusarium varieties Golets, Tubinsky and Zolotoi pochatok. In this way, in vitro, oat varieties were tested for resistance to toxic metabolites of two types of micromycetes $F$. eqiseti and F. poae. 